\title{
Mean skin temperature estimated from 3 measuring points can predict sleeping thermal sensation
}

\author{
Lan, Li; Xia, Lulu; Tang, Jieyu; Wyon, David Peter; Liu, Hua
}

Published in:

Building and Environment

Link to article, DOI:

10.1016/j.buildenv.2019.106292

Publication date:

2019

Document Version

Peer reviewed version

Link back to DTU Orbit

Citation (APA):

Lan, L., Xia, L., Tang, J., Wyon, D. P., \& Liu, H. (2019). Mean skin temperature estimated from 3 measuring points can predict sleeping thermal sensation. Building and Environment, 162, [106292].

https://doi.org/10.1016/j.buildenv.2019.106292

\section{General rights}

Copyright and moral rights for the publications made accessible in the public portal are retained by the authors and/or other copyright owners and it is a condition of accessing publications that users recognise and abide by the legal requirements associated with these rights.

- Users may download and print one copy of any publication from the public portal for the purpose of private study or research.

- You may not further distribute the material or use it for any profit-making activity or commercial gain

- You may freely distribute the URL identifying the publication in the public portal 


\title{
Mean skin temperature estimated from 3 measuring points can predict sleeping thermal sensation
}

\author{
Li Lan ${ }^{1 *}$, Lulu Xia ${ }^{1}$, Jieyu Tang ${ }^{1}$, David P. Wyon ${ }^{2}$, Hua Liu ${ }^{3,4 *}$ \\ ${ }^{1}$ Department of Architecture, School of Design, Shanghai Jiao Tong University, Shanghai \\ 200240, China. \\ ${ }^{2}$ International Centre for Indoor Environment and Energy, Department of Civil Engineering, \\ Technical University of Denmark (DTU), DK-2800 Kgs. Lyngby, Denmark. \\ ${ }^{3}$ State key laboratory of Air Conditioning Equipment and System Energy Conservation, Zhuhai \\ 519070, China. \\ ${ }^{4}$ Gree Electric Appliances Inc., Zhuhai 519070, China. \\ Li Lan, E-mail: 1anli2006@sjtu.edu.cn; Hua Liu, E-mail: Liuhua@cn.gree.com
}

\begin{abstract}
Sensory input from the skin appears to be of crucial importance in the regulation of sleep but there has been limited research on human skin temperature during sleep. The present study was undertaken to validate calculation of the mean skin temperature (MST) of sleeping subjects from measurements at only three locations: forehead, chest, and foot, by means of an analysis of skin temperature data obtained from four human experiments that investigated the effects of thermal environment on the sleep quality and thermal comfort of a total of 64 sleeping subjects. The results show that skin temperatures were more evenly distributed across the body surface of sleeping subjects than they are when subjects are awake. The skin temperature of the forehead was highly correlated with thermal sensation in long-term measurements. MST values calculated using the three-point method were found to predict thermal sensation while sleeping better than those calculated using the normally accepted Hardy and Dubois's seven-point method. The validity, convenience and reliability of this approach, which also causes less sleep disturbance, makes it a suitable choice for obtaining estimates of mean skin temperature in sleep studies.
\end{abstract}


Keywords: skin temperature; mean skin temperature; MST; thermal comfort; thermal sensation; sleep

\section{Introduction}

Sleep is essential for the body to recover from both physical and psychological fatigue suffered throughout the day and is important for human health [1]. The thermal environment is one of the primary causes of sleep disturbance [2,3], which can also be caused by such factors as health, emotional state and bedding. Exposure to moderate heat or cold results in increased sleep onset latency and decreased slow wave sleep (SWS) [3]. Even mild heat exposure leads to significantly decreased sleep quality in older people [4]. Creating a thermally comfortable sleeping environment is thus an important research topic.

Input from the skin appears to be of crucial importance in the regulation of sleep [5]. Since the human skin forms the interface between the human body and the thermal environment, skin temperature provides essential information on heat loss from the human body. Moreover, when we feel warm or cool, we do not actually sense the air temperature, but rather the temperature of the thermoreceptors, most of which are located in the periphery of the body and in or around the great veins in the upper abdomen and thorax, as they send signals to the hypothalamus at the base of the brain when stimulated [6]. The hypothalamus will then trigger autonomic thermoregulation to help the human body maintain a constant central body temperature in a hot or cold environment. Skin temperature has been shown to have the same weighting as core temperature for predicting thermal sensation in uniform conditions [6]. Importantly, the hypothalamus not only controls thermoregulation, but also regulates sleep. It has been suggested that sleep is regulated by opposing wake-promoting and sleep promoting mechanisms located in the hypothalamus [5]. Skin warming induced by cutaneous vasodilation is likely to contribute to the activation of thermosensitive sleep-active neurons in the hypothalamus [7]. The effect of skin warming on sleep was confirmed in a human subject experiment, which showed that an experimentally induced but subtle increase in skin temperature, within the thermoneutral range, caused a decrease in sleep-onset latency [8]. 
Human subject experiments also found that mean skin temperature (MST) changed as a function of the thermal environment during the sleeping period $[3,9,10]$. These results suggest that skin temperature is of primary importance in assessing the thermoregulation and sleep regulation of sleeping subjects.

If the skin temperature plays such an important role in sleep regulation, it should be monitored together with sleep quality in laboratory, clinical and home situations. Various formulas have been proposed for measuring specific sites of the body and assigning corresponding weighting factors to estimate the MST in subjects who are awake. The 15 sites recommended by Winslow et al. appear to constitute a reasonable maximum [11].The Hardy and Dubois seven-point formula remains one of the best means for weighting the relative areas of each of the regions [12], and is commonly used as criterion for the appraisal of thermal comfort [13-16]. A weighting system for the calculation of MST from four-point measurement is also often used, probably because of its acceptable reliability and convenience [17]. Liu et al. evaluated different weighting formulas for calculating the MST, reporting that a 10-site method was the most appropriate method in human thermal comfort studies [18]. Mitchell and Wyndham compared different methods for calculating MST and found that the unweighted mean temperature at 15 sites is appropriate for accurate work [19]. While awake, a precondition for thermal comfort is maintaining MST in the range $33-34{ }^{\circ} \mathrm{C}$ when sedentary, and at a lower temperature when engaged in higher levels of activity [20,21]. While asleep, it is important to be able to measure skin temperature not only reliably over a long sleeping period, but also without causing sleep disturbance. Skin temperature should thus be measured at as few sites as possible. To our knowledge, no research on the measurement method of skin temperature in sleeping subjects has yet been published. Based on the skin temperatures measured with Hardy and Dubois seven-point method in four human subject experiments, the distribution of skin temperature over the human body and the correlation between skin temperatures and sleeping thermal sensation were analysed and are reported in the present study. A new weighting method for calculating the MST of sleeping subjects from measurements at only 3 sites was proposed and validated. This makes it possible to accurately predict the thermal state of sleeping subjects and minimises the resulting disturbance of sleep quality by the measurement process. 


\section{Methods}

\subsection{The human subject experiments}

Table 1 summarizes the nature of the four experiments from which data were taken, all of which investigated the effects of the thermal environment on sleep quality [9, 22-24]. Although different thermal interventions were performed, a similar experimental approach was used in each of these experiments. Healthy human subjects without sleep disorders were recruited for the experiments, using a within-subject design. All of the protocols were approved by the university's ethics committee and conformed to the guidelines in the Declaration of Helsinki. Verbal and written informed consent were obtained from each subject prior to their participation in each experiment.

Table 1 Experimental studies that measured the skin temperature of sleeping subjects

\begin{tabular}{|c|c|c|c|c|}
\hline Source & Thermal interventions & Bed covering & Subjects & Main conclusions \\
\hline Lan et & Constant environment. Three air & A mattress bed and a & 18 & Moderate heat or cold \\
\hline al., 2014 & temperatures $\left(23^{\circ} \mathrm{C}, 26^{\circ} \mathrm{C}\right.$, & double-layered & healthy & exposure results in \\
\hline \multirow[t]{3}{*}{ [9] } & $30^{\circ} \mathrm{C}$ ) were used to create cool, & cotton sheet, with an & adults & increased sleep onset \\
\hline & neutral, and warm sleep & estimated insulation & & latency and decreased \\
\hline & environments. & of 1.64 clo. & & SWS. \\
\hline Lan et & Constant vs. dynamic & A mattress bed and a & 12 & No significant differences \\
\hline al., 2016 & environment. A constant & double-layered & healthy & in sleep quality were \\
\hline \multirow[t]{6}{*}{ [22] } & temperature reference condition & cotton sheet, with an & adults & found when the air \\
\hline & $\left(26^{\circ} \mathrm{C}\right)$, a Fall-Rise change & estimated insulation & & temperature changed in \\
\hline & condition $\left(28^{\circ} \mathrm{C}-27^{\circ} \mathrm{C}-26^{\circ} \mathrm{C}-\right.$ & of 1.64 clo. & & the range $26^{\circ} \mathrm{C}-28^{\circ} \mathrm{C}$. \\
\hline & $27^{\circ} \mathrm{C}-28^{\circ} \mathrm{C}$ ), and a Rise-Fall & & & \\
\hline & change condition $\left(25^{\circ} \mathrm{C}-26^{\circ} \mathrm{C}-\right.$ & & & \\
\hline & $\left.27^{\circ} \mathrm{C}-28^{\circ} \mathrm{C}-27^{\circ} \mathrm{C}-26^{\circ} \mathrm{C}\right)$ & & & \\
\hline Lan et & Constant vs. dynamic & A mattress bed and a & 18 & The subjects took a \\
\hline al., 2016 & environment. Air temperature & double-layered & healthy & longer time to fall asleep \\
\hline [23] & was maintained at a lower but & cotton sheet, with an & adults & when the environment \\
\hline
\end{tabular}




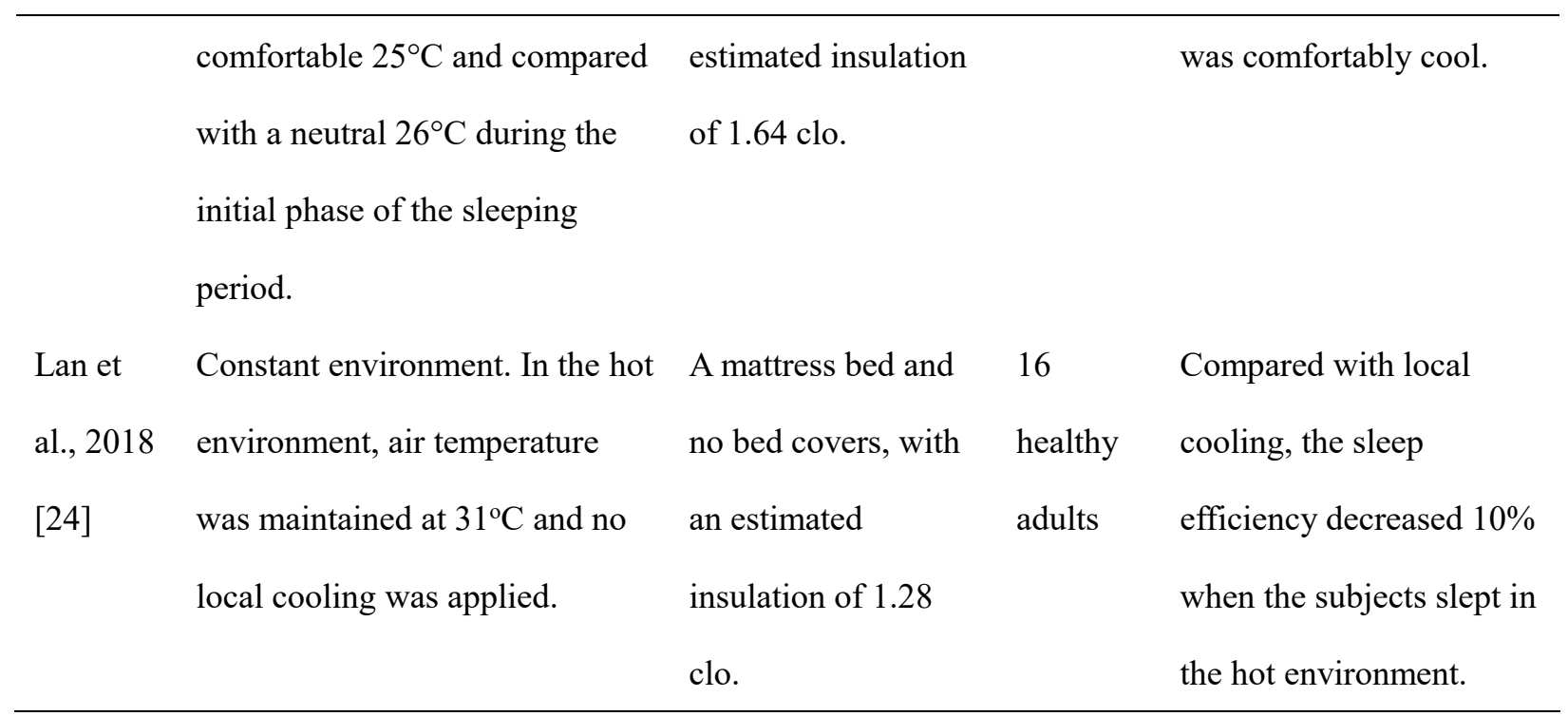

Measurements. Sleep quality was evaluated subjectively, using questionnaires completed in the morning, and objectively, by analysis of electroencephalogram (EEG), electrooculogram (EOG) and electromyogram (EMG) signals that were continuously monitored during the sleeping period. A 7-point scale (-3-cold, -2-cool, -1-slightly cool, 0-neutral, 1-slightly warm, 2-warm, 3-hot) was used to report thermal sensation.

The skin temperatures at seven sites were continuously measured during the sleeping period, including the forehead, chest, posterior forearm, hand, anterior thigh, anterior calf, and foot, as specified in the Hardy and Dubois method [12]. Skin temperature at these seven locations has often been reported, permitting us to compare differences in skin temperature between subjects who were asleep and awake. As far as we know, this seven-point is also the maximum number of skin temperature measurements of sleeping subjects that has been reported in the literature. The MST was calculated as the weighted mean of local skin temperatures [12]:

$M S T=0.07 t_{s k, f h}+0.35 t_{s k, c}+0.14 t_{s k, p f}+0.05 t_{s k, h}+0.19 t_{s k, a t}+0.13 t_{s k, a c}+0.07 t_{s k, f}$

where tsk,fh is the skin temperature of the forehead; tsk,c is that of the chest; tsk,pf is that of the posterior forearm; tsk,h is that of the hand; tsk,at is that of the anterior thigh; tsk,ac is that of the anterior calf; and tsk, $\mathrm{f}$ is that of the foot.

Experimental procedure (Figure 1). Subjects arrived at the waiting room (which was adjacent 
to their sleeping chambers and was maintained at a thermally neutral temperature) at 21:00, rested for $30 \mathrm{~min}$ in order to adapt thermally, and completed the Profile Of Mood States (POMS) questionnaire (Q1) in the next 10 min. The physiological parameter sensors were attached in the course of the next 40 minutes. The subjects then entered their sleeping chamber and after lying on the bed for 30 minutes, recorded their thermal comfort (Q2), which took 10 minutes. Sleep could then begin at 23:00 when the lights were turned off. The physiological parameters were continuously measured throughout the night until subjects were woken up at 07:00 the next morning. The subjects then completed the sleep quality questionnaires (Q3) and recalled their thermal sensation during the night (Q2).

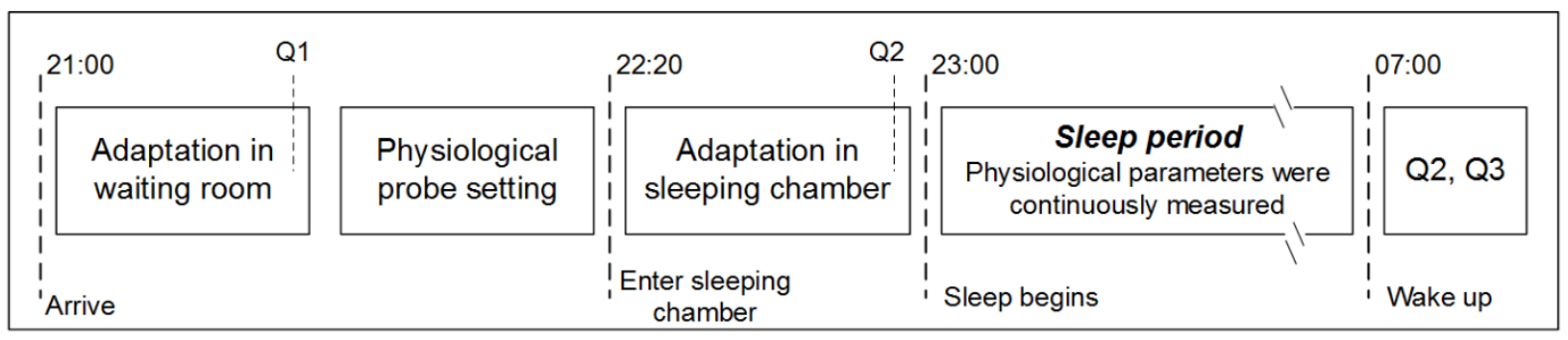

Figure 1 Experimental procedures for an all-night sleep; Q1- Profile of mood states (POMS) questionnaire, Q2- thermal sensation and thermal comfort questionnaire, Q3- sleep quality questionnaire

\subsection{Data analysis}

The data were tested for normality using the Shapiro-Wilk's W test. Normally distributed data were subjected to analysis of variance in a repeated measures design and a Paired Samples t-test. Huynh-Feldt statistics were used to adjust the violation of sphericity. Not-normally distributed data were analysed using Friedman analysis of variance and the Wilcoxon MatchedPairs Signed-Ranks test. The significance level was set to $0.05(P<0.05)$.

Only skin temperatures measured in the thermally neutral conditions of the 4 experiments were compared with a reference dataset taken from 3 independent studies of subjects who were also thermally neutral but awake $[6,25,26]$. Only skin temperatures measured in uniform and constant thermal environments were used to calculate the averaged MST for the whole night, which were then linearly correlated with thermal sensation. However, skin temperatures 
measured in all the conditions of the 4 experiments (except those in which local cooling was applied) were used to validate the proposed three-point estimate of MST.

As noted above, the subjects were asked to recall their thermal sensation during the previous night's sleep. Regression analysis was performed between the skin temperatures and these recalled thermal sensation votes.

\subsection{A three-point weighting approach}

A three-point weighted mean approach to the estimation of MST as a predictor of thermal comfort sensation while asleep was proposed. Three locations including forehead, chest, and foot were measured, assigning the weighting factors of $40 \%, 40 \%$, and $20 \%$, respectively. As explained below in Table 3, these weighting factors were derived by taking account not only of the relative surface areas represented by each measurement, but also of the sensitivity of thermal sensation to skin temperature at each point. The proposed formula for computing MST from 3 measurement sites is thus:

$M S T=0.4 \times t_{s k, f h}+0.4 \times t_{s k, c}+0.2 \times t_{s k, f}$

The derivation of this three-point approach was as follows.

\subsubsection{Selection of measuring points}

The skin temperatures measured at the seven body positions and the mean skin temperature while sleeping or awake in a thermally neutral environment were compared, as shown in Figure 2. While sleeping, a $1.5^{\circ} \mathrm{C}$ difference was observed between the coldest area (the arm) and the warmest area (the chest), with a variance of 0.21 among the seven positions. While awake, the difference between the highest and lowest skin temperature was much larger, $2 .{ }^{\circ} \mathrm{C}$ in Olesen's (1984) study [25], $3.1^{\circ} \mathrm{C}$ in Zhang's study [6], and $3.7^{\circ} \mathrm{C}$ in Houdas's (1982) study [26]. The variance of skin temperature over the seven body positions was consistently larger: $0.87^{\circ} \mathrm{C}$, $1.09^{\circ} \mathrm{C}$ and $1.52^{\circ} \mathrm{C}$ in the three studies, respectively. While awake, the warmest skin areas were the head and chest regions, as was the case when sleeping, but the coldest areas were the lower extremities (leg and foot) instead of the arms. Under neutral conditions, the averaged MST while sleeping was approximately $34.6^{\circ} \mathrm{C}$, which is $1{ }^{\circ} \mathrm{C}$ higher than while awake. The results 
show that skin temperatures were more evenly distributed over the human body when sleeping, suggesting that the mean skin temperature can be estimated with fewer measurement points, which would reduce the sleep disturbance caused by the presence of multiple skin temperature sensors.

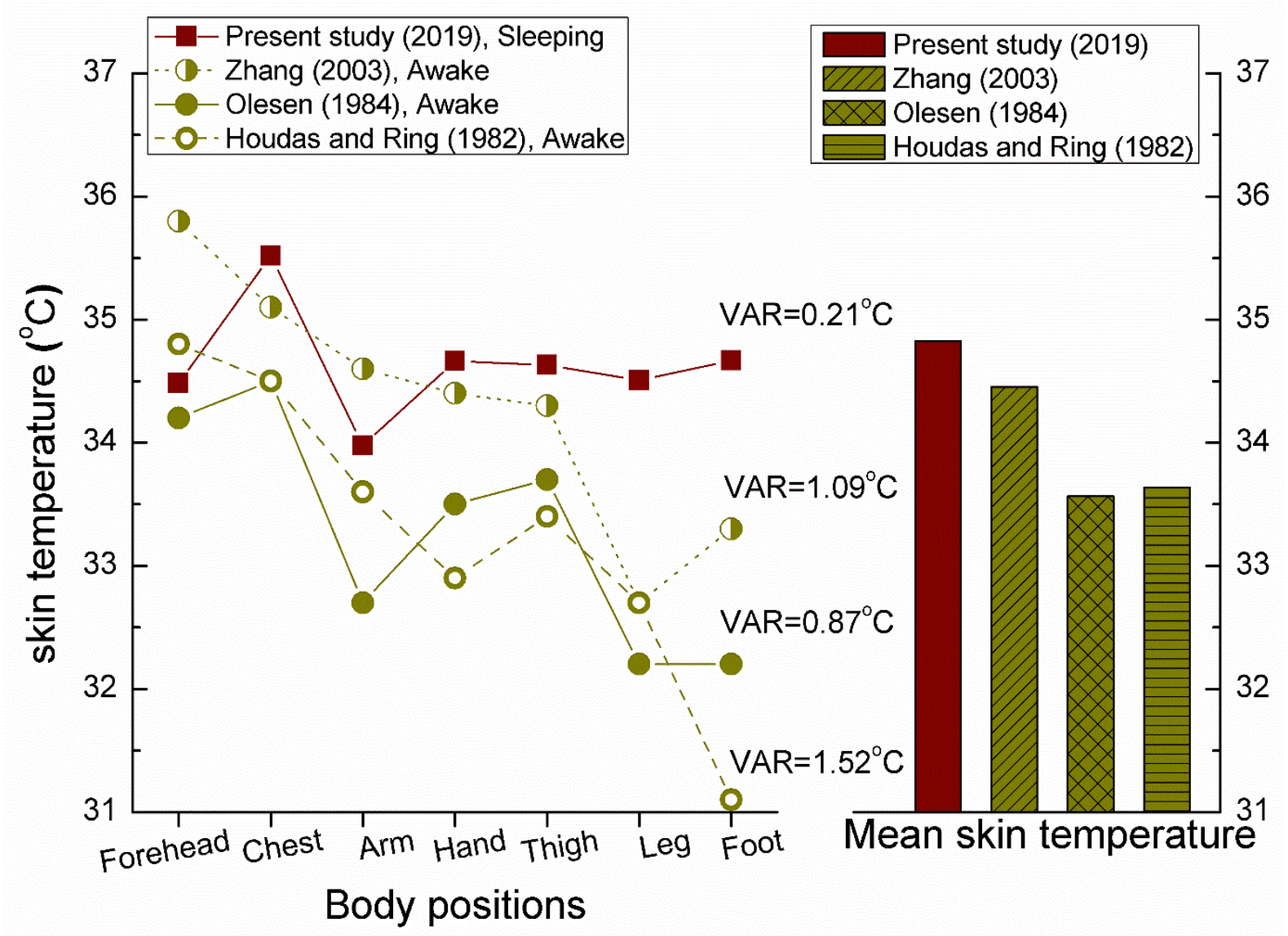

Figure 2 Distribution of skin temperature and mean skin temperature while sleeping or awake in a thermally neutral environment. VAR was the variance of skin temperatures measured at the seven body positions in each study.

McIntyre reported that whole-body thermal sensation was roughly a linear function of mean skin temperature between $29^{\circ} \mathrm{C}$ and $34^{\circ} \mathrm{C}$ [13], and this was confirmed in later studies [18]. Linear regression analysis was performed between the skin temperatures of the seven body parts and (recalled) whole-body thermal sensation during sleep. The separation of head, trunk, and extremity regions followed Zhang's study [6]. The regression results summarized in Table 2 show that the skin temperature of the forehead correlated linearly with thermal sensation, with 
a very high R-square value, accounting for $96 \%$ of the variance. The skin temperatures of other parts of the body were correlated with thermal sensation with moderate R-square values, except the skin temperature of the hand, which was not linearly correlated with thermal sensation.

Table 2 Linear regression analysis between skin temperature (ST) and whole-body thermal sensation

\begin{tabular}{lllll}
\hline Regions & Dependent variable (Y) & Equation $^{\mathfrak{L}}$ & $\mathrm{R}$ & Sig. \\
\hline Head & ST at forehead, & $\mathrm{Y}=0.286 \times \mathrm{TSV}+34.538$ & 0.966 & $\mathrm{P}<0.001^{* * *}$ \\
& $33.0^{\circ} \mathrm{C} \leq \mathrm{Y} \leq 35.7^{\circ} \mathrm{C}$ & & & \\
Trunk & $\mathrm{ST}$ at chest, & $\mathrm{Y}=0.191 \times \mathrm{TSV}+35.172$ & 0.578 & $\mathrm{P}<0.05^{*}$ \\
& $33.4^{\circ} \mathrm{C} \leq \mathrm{Y} \leq 36.8^{\circ} \mathrm{C}$ & & & \\
Extremities & $\mathrm{ST}$ at arm, & $\mathrm{Y}=0.262 \times \mathrm{TSV}+33.832$ & 0.800 & $\mathrm{P}<0.01^{* *}$ \\
& $31.9^{\circ} \mathrm{C} \leq \mathrm{Y} \leq 35.9^{\circ} \mathrm{C}$ & & & \\
& $\mathrm{ST}$ at hand, & $\mathrm{Y}=0.101 \times \mathrm{TSV}+34.549$ & 0.351 & $\mathrm{NS}$ \\
& $32.1^{\circ} \mathrm{C} \leq \mathrm{Y} \leq 35.7^{\circ} \mathrm{C}$ & & & \\
& $\mathrm{ST}$ at thigh, & $\mathrm{Y}=0.215 \times \mathrm{TSV}+34.324$ & 0.587 & $\mathrm{P}<0.05^{*}$ \\
& $32.9^{\circ} \mathrm{C} \leq \mathrm{Y} \leq 36.0^{\circ} \mathrm{C}$ & & & \\
$\mathrm{ST}$ at leg, & & $\mathrm{Y}=0.248 \times \mathrm{TSV}+34.172$ & 0.594 & $\mathrm{P}<0.05^{*}$ \\
$32.8^{\circ} \mathrm{C} \leq \mathrm{Y} \leq 36.1^{\circ} \mathrm{C}$ & & & \\
$\mathrm{ST}$ at foot & & $\mathrm{Y}=0.152 \times \mathrm{TSV}+34.536$ & 0.626 & $\mathrm{P}<0.05^{*}$ \\
$32.7^{\circ} \mathrm{C} \leq \mathrm{Y} \leq 35.9^{\circ} \mathrm{C}$ & & & \\
\hline
\end{tabular}

${ }^{\mathfrak{f}} \mathrm{Y}=\mathrm{A} \times \mathrm{TSV}+\mathrm{B}$. Independent variable-- whole-body thermal sensation votes (TSV): $3 \leq \mathrm{TSV} \leq+3$, cold $(-3)$, cool $(-2)$, slightly cool $(-1)$, neutral $(0)$, slightly warm $(+1)$, warm $(+2)$, hot $(+3)$;

$* \mathrm{P}<0.05, * * \mathrm{P}<0.01, * * * \mathrm{P}<0.001, \mathrm{NS}-$ Not Significant $(\mathrm{P}>0.05)$

We thus proposed to measure skin temperatures at the three sites, i.e.., forehead, chest, and foot, which represent three regions of human body (the head, trunk, and extremities) and are the most important body parts for heat exchange between the body and the environment, and 
for thermoregulation while sleeping. The skin temperature of the forehead was included because the forehead is a highly thermosensitive area [27] and its skin temperature is highly correlated with thermal sensation (Table 2). The chest area accounts for a very large surface area and thus has a major effect on the mean skin temperature of the whole body. The skin temperature of the foot was selected because it has been found to be important for good sleep, a comparatively higher foot skin temperature being able to promote a rapid onset of sleep [28]. Skin temperatures measured at the above three sites are therefore expected to reflect the thermal state of sleeping subjects and to correlate closely with sleep quality.

\subsubsection{Determination of weighting factors}

In Hardy and Dubois method, the weighting of each point was determined based on their relative skin surface area. In addition to skin surface area, the present study took the sensitivity of each site into account when determined the weighting factors. The $\mathrm{R}$ square of each correlation between skin temperature and thermal sensation as shown in Table 2 was used to determine skin sensitivity, and the proportion of skin surface area of each region was determined according to [29]. Table 3 shows the calculation of the weighting factors for the three sites, which was determined to be $0.4,0.4$ and 0.2 for forehead, chest, and foot, respectively, by rounding down to one decimal place.

Table 3 Determination of weighting factor for each position.

\begin{tabular}{lllllll}
\hline & $\begin{array}{l}\text { Sensitivity } \\
\left(\mathrm{S}_{1}=\mathrm{R}\right.\end{array}$ & $\begin{array}{l}\text { Skin surface } \\
\text { area }\left(\mathrm{S}_{2}\right)^{\S}\end{array}$ & $\mathrm{a}=\mathrm{S}_{1} / \sum \mathrm{S}_{1}$ & $\mathrm{~b}=\mathrm{S}_{2} / \sum \mathrm{S}_{2}$ & $(\mathrm{a} \times \mathrm{b}) / \sum(\mathrm{a} \times \mathrm{b})$ & $\begin{array}{l}\text { Weighting } \\
\text { factor }^{\dagger}\end{array}$ \\
\hline square $)^{£}$ & & & & & \\
\hline Forehead & 0.966 & 0.076 & 0.445 & 0.269 & 0.375 & 0.4 \\
Chest & 0.578 & 0.136 & 0.266 & 0.482 & 0.401 & 0.4 \\
Foot & 0.626 & 0.070 & 0.288 & 0.248 & 0.224 & 0.2 \\
Sum $\sum$ & 2.170 & 0.282 & $/$ & $/$ & $/$ & \\
\hline
\end{tabular}

${ }^{£} \mathrm{~A}$ is the $\mathrm{R}$ square of each correlation between skin temperature and thermal sensation as shown in Table 2.

$\S$ The proportion of skin surface area was determined according to [29]. 
${ }^{\dagger}$ The weighting factors were determined by rounding down to one decimal place of $(\mathrm{a} \times \mathrm{b}) / \Sigma$ $(\mathrm{a} \times \mathrm{b})$.

\section{Validation of the three-point approach}

In the four human subject experiments listed in Table 1, skin temperatures at seven sites on the body were measured and calculated according to the Hardy and Dubois method [12]. In the present paper, we suggest that only three of the seven measurement sites need be used to predict thermal sensation when sleeping. Averaged MSTs for the whole night calculated from only three sites were compared with those calculated using the Hardy and Dubois seven-point method, as shown in Figure 3. It may be seen that the MSTs calculated using the two methods are in good agreement, differing by less than $0.6^{\circ} \mathrm{C}$ in almost all conditions.

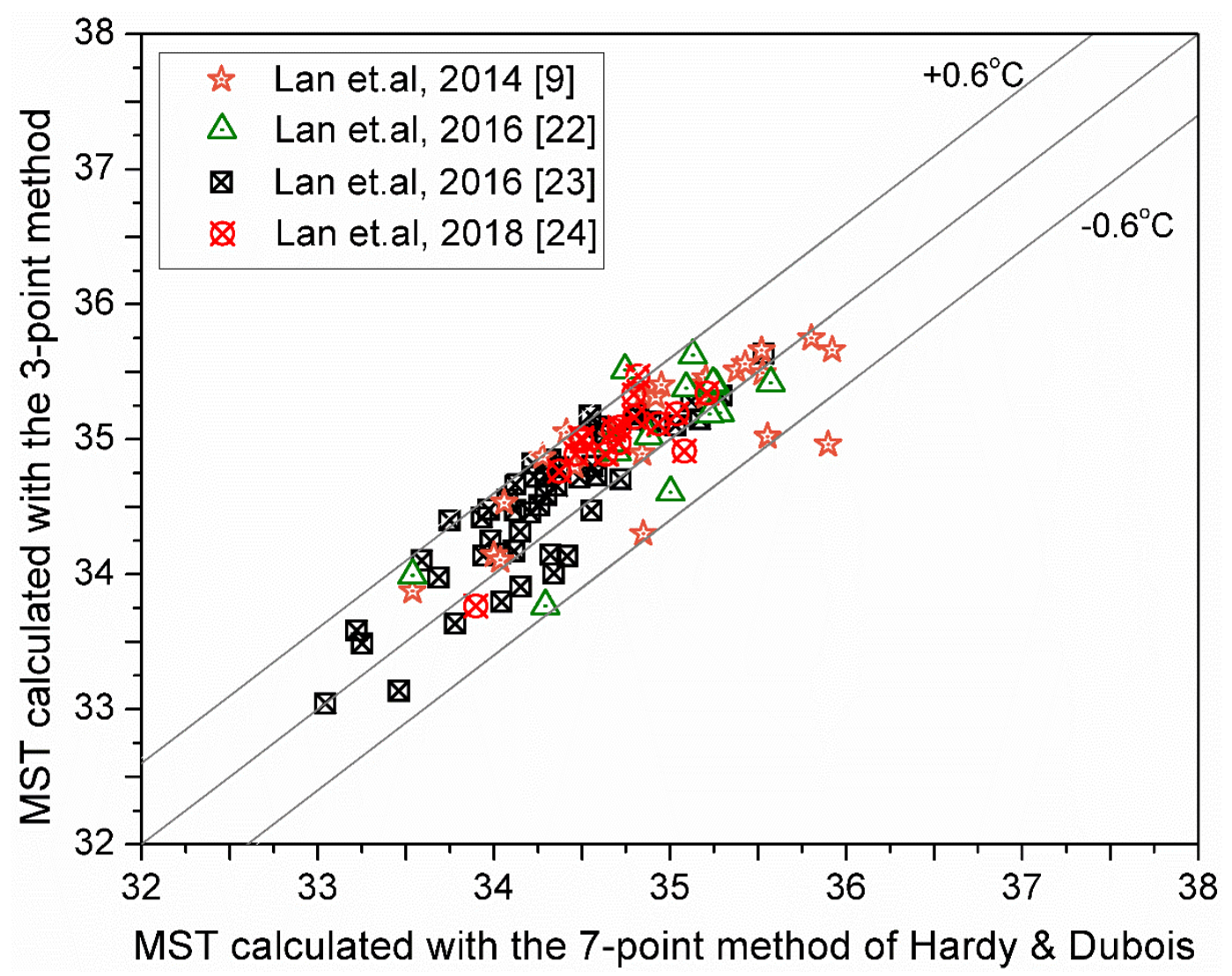

Figure 3 Comparison of mean skin temperatures (MSTs) calculated with the Hardy and Dubois 7-point formula and the proposed 3-point formula 
In the four human subject experiments, after getting up in the morning, the subjects were asked to recall their thermal sensation during the preceding night on a 7-point scale (-3-cold, 2-cool, -1-slightly cool, 0-neutral, 1-slightly warm, 2-warm, 3-hot). Both averaged MSTs of whole night calculated with the three-point method and the seven-point method were significantly correlated with the reported thermal sensation: both increased with higher thermal sensation vote, as expected (Figure 4). The systematic decrease in the slope of the curve in this figure as thermal comfort sensation increases may be attributed to progressively increasing evaporative heat loss in warmer conditions, as increased evaporation of sweat necessarily decreases skin temperature. A better correlation was obtained between the MST calculated with the three-point method and the thermal sensation vote, with a R-square value of 0.89 . Thus, compared with the seven-point method, the three-point method improves the correlation between MST and thermal sensation.

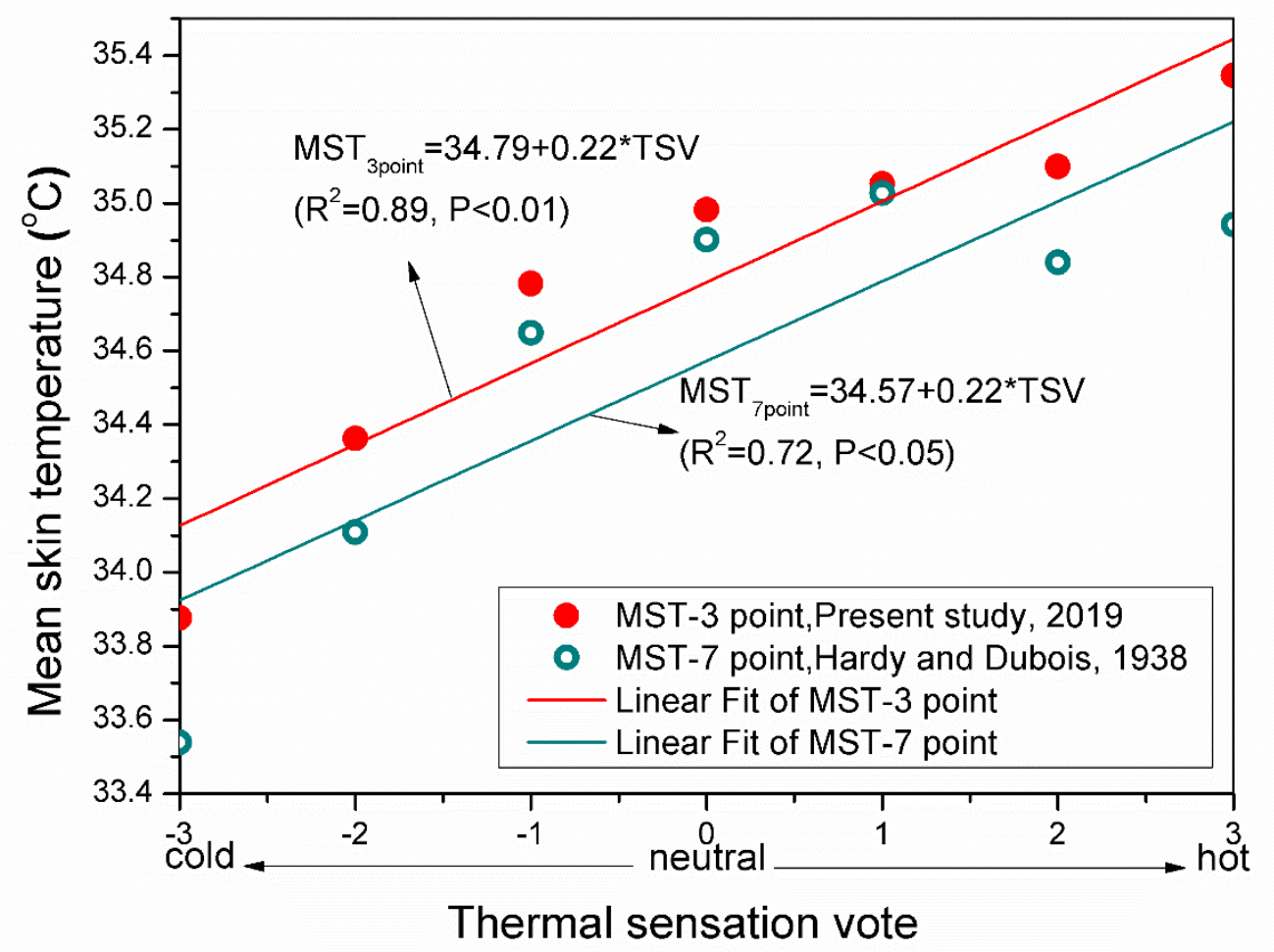

Figure 4 Thermal sensation votes (TSV) as a function of mean skin temperatures (MSTs) calculated with the Hardy and Dubois 7-point method and the proposed 3-point method

Figure 5 shows the variation of the MSTs of 18 sleeping subjects, as calculated using the Hardy 
and Dubois seven-point method, and the proposed three-point method, throughout the night when the air temperature was kept being constant at about $26^{\circ} \mathrm{C}$ [23]. After lights off, a similarly increasing trend was observed in the MSTs calculated with the two methods. This increase reached its peak after about $60 \mathrm{~min}$. It then decreased gradually and began to fluctuate with small variations. The very small fluctuations that occurred during the greater part of the sleep period suggest that it is acceptable to use the averaged MST for the whole night for assessment of the thermal state of the body in a constant thermal environment.

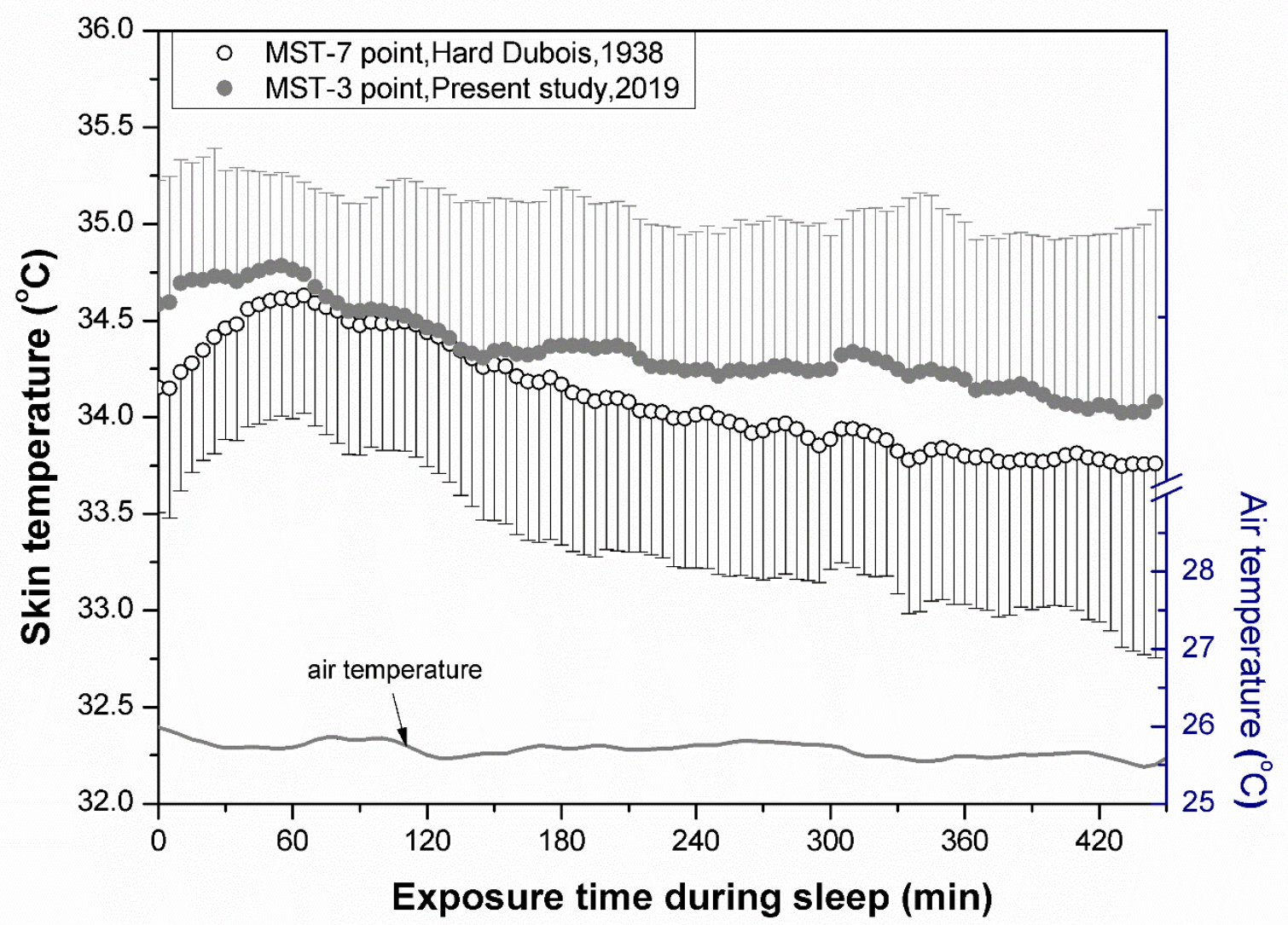

Figure 5 Variation of mean skin temperatures (MSTs) throughout the night when the air temperature was maintained constant at $26^{\circ} \mathrm{C}$, using the data from Lan et al. [23]. (Error bars represent the standard deviation of the mean (STD))

Figure 6 shows the variation of the MSTs of 18 sleeping subjects, as calculated using the Hardy and Dubois seven-point method, and the proposed three-point method, in exposures in which the air temperature changed in a Rise-Fall pattern $\left(25^{\circ} \mathrm{C}-26^{\circ} \mathrm{C}-27^{\circ} \mathrm{C}-28^{\circ} \mathrm{C}-27^{\circ} \mathrm{C}-26^{\circ} \mathrm{C}\right)$ 
[23]. Again, a similarly variation was observed in the MSTs calculated with the two methods. The MST increased after lights off, reaching its peak after about $60 \mathrm{~min}$, and then decreased gradually, although the decrease of skin temperature was greatly suppressed by the increasing air temperature. Compared with the seven-point method, it may be seen that the MST calculated with the three-point method varied more in accordance with the increase of air temperature, from 180 min to $300 \mathrm{~min}$, during which no significant increase in the MST calculated with the seven-point method was observed. At around $330 \mathrm{~min}$, both MST values stopped decreasing even though the air temperature continued to decrease. This seems likely to have been due to an increase in metabolic rate towards the end of the sleeping period, as the subjects began to wake up. Overall, the MST calculated with the three-point formula corresponded very well to the change of air temperature.

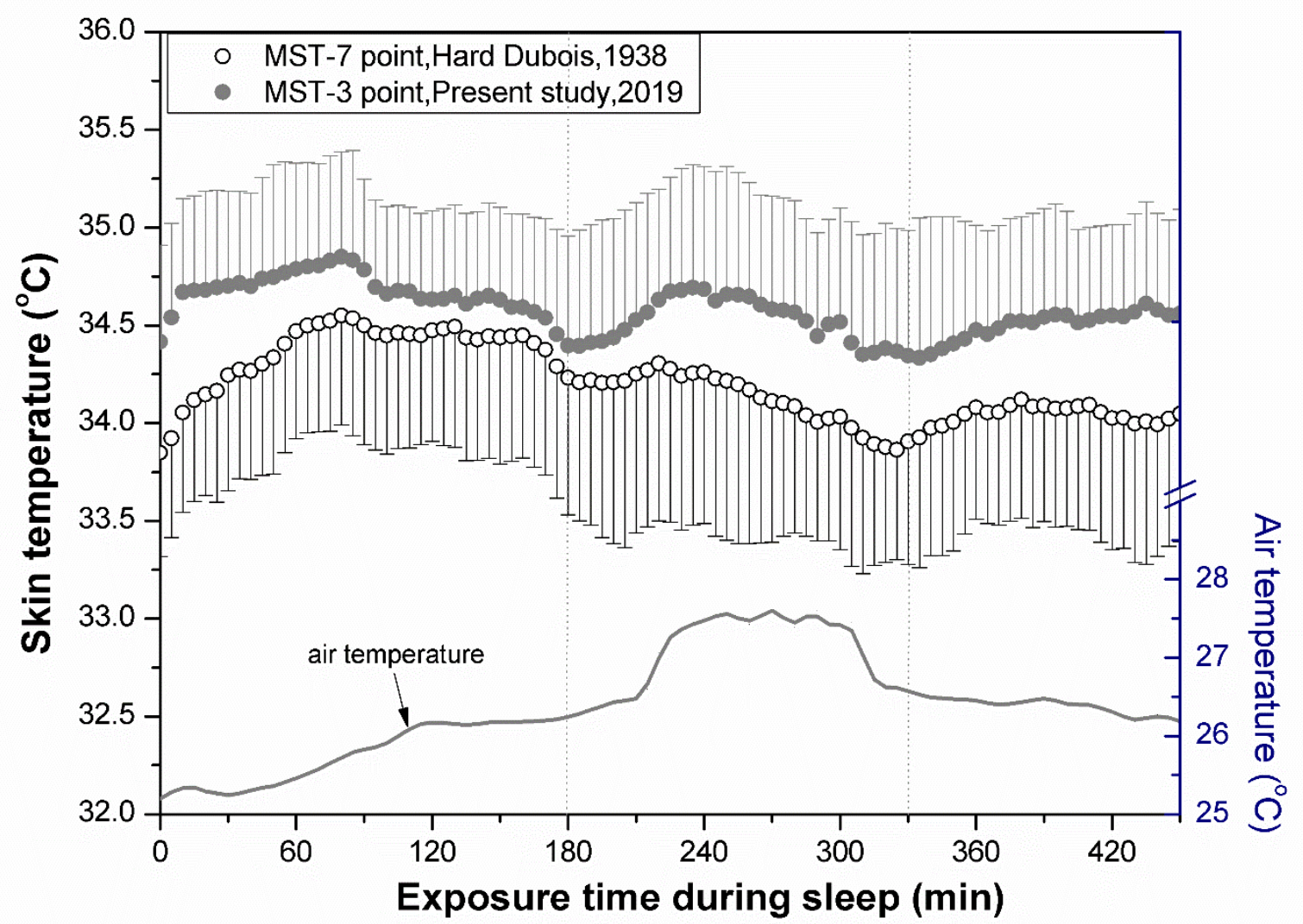

Figure 6 Variation of mean skin temperatures (MSTs) with change of air temperature $\left(25^{\circ} \mathrm{C}\right.$ $26^{\circ} \mathrm{C}-27^{\circ} \mathrm{C}-28^{\circ} \mathrm{C}-27^{\circ} \mathrm{C}-26^{\circ} \mathrm{C}$ ), using the data from Lan et al. [23]. (Error bars represent the standard deviation of the mean (STD)) 
To investigate why the MST calculated with the three-point method varied more in accordance with the increase of air temperature, the skin temperatures measured at the seven body positions throughout the night are shown in Figure 7. It may be seen that the skin temperature of the head and distal part, including the hand and foot were more sensitive to the change of air temperature, while those of the thigh and leg were the least influenced by changes in the air temperature.
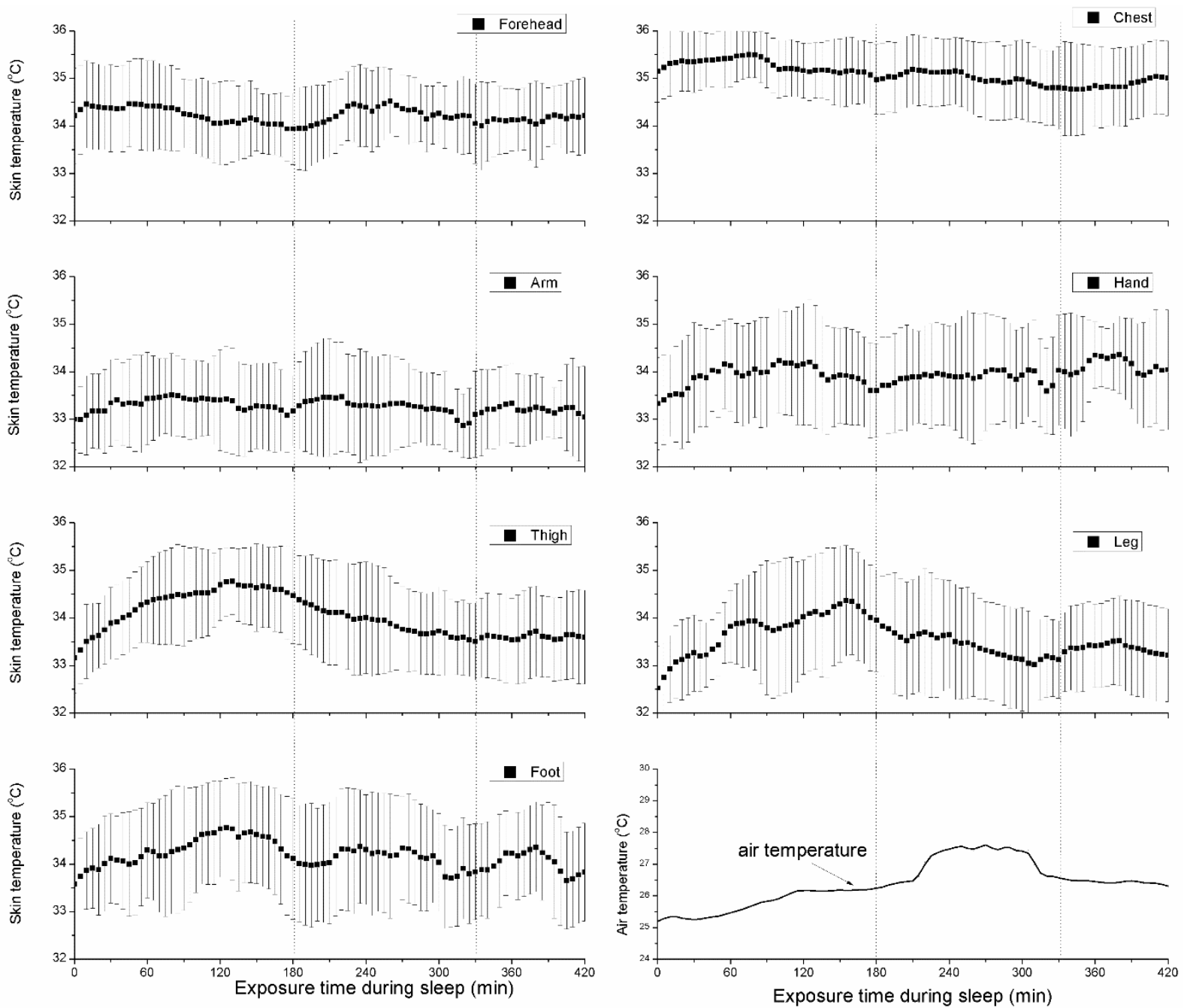

Figure 7 Variation of skin temperatures measured at the seven body positions with change of air temperature $\left(25^{\circ} \mathrm{C}-26^{\circ} \mathrm{C}-27^{\circ} \mathrm{C}-28^{\circ} \mathrm{C}-27^{\circ} \mathrm{C}-26^{\circ} \mathrm{C}\right)$, using the data from Lan et al. [23]. (Error bars represent the standard deviation of the mean (STD))

\section{Discussion}

The present study documents that skin temperatures were more evenly distributed across the body in sleeping subjects than has been reported for subjects who were awake (Fig. 2). This 
difference may be due to the different body posture, the relaxation of the body (reduction of the sympathetic vasoconstrictor tonus), and the use of bed coverings. This more even distribution makes it possible to estimate MST from measurements of skin temperature at fewer sites on sleeping subjects, which causes less sleep disturbance. In view of this more even distribution of skin temperature, we propose that the measurement sites for estimating mean skin temperature while asleep should be reduced from seven points to three points, i.e., the forehead, chest, and foot. These three sites represent the head area, trunk, and extremities of the human body. The detailed reason for measuring the skin temperature of the forehead is explained in the next paragraph. The trunk area accounts for most of the human body surface area, so chest skin temperature plays a significant role in thermoregulation. The skin temperature of the foot was included because a previous study had found that foot skin temperature is important for good sleep, and that keeping the feet warm promoted the rapid onset of sleep [25]. In winter, a person with cold feet is unlikely to fall asleep quickly even if all other body parts are not cold. Despite the limited surface area, the distal skin temperature appears to be more important than the proximal skin temperature for sleep regulation [3], and this was confirmed in the present study (Figure 7), so it is necessary to include a distal skin temperature such as the foot. A less theoretical but practically important reason for selecting the proposed three sites was that we observed that the sensors on these three sites continued to be firmly attached to the skin whereas the sensors on the arm, leg, and thigh were observed to become detached from the skin more frequently. During sleep, unintentional scratching of the latter three parts of the body may have occurred, resulting in the detachment of sensors. The duration of normal human sleep is relatively long, lasting about $8 \mathrm{~h}$. To obtain a full description of the thermal condition of the human body, it is necessary to measure the skin temperature throughout the entire sleeping period.

To date, few researchers have focused on the contribution of thermal comfort to sleep quality and a limited number of studies measured the skin temperature of sleeping subjects $[4,9,10$, 22-24]. However, only the calculated MST was reported in these studies, instead of each skin temperature, so data from these experiments could not be used to validate the proposed threepoint approach. Compared with measurement at seven points, three-point measurement 
improved the correlation between MST and thermal sensation (Fig. 4) in the present data. When examining the variation of MST with change of air temperature during the sleeping period, the MST calculated with the three-point method varied more with changes in air temperature (Figure 6). The reason could be that the more influential parts, including head and foot, were assigned higher weighting factors in the three-point than the seven-point method. In the proposed three-point method, the weighting factor of each body site was derived not only from its skin surface area, but also from its relative sensitivity to thermal sensation (Table 3). For thermal comfort prediction, it is better to take the thermal influence of each body part into account when calculating MST, although the weighting factors proposed in the present study should be further confirmed by more studies. Given that the three-point method has the advantage of more convenient application of sensors and causes less sleep disturbance, it is recommended for the estimation of the mean skin temperature of sleeping subjects for the purpose of determining their thermal sensation in a uniform environment. In a uniform and not extreme environment, it may be enough to assess the whole-body thermal sensation, as we did in the four studies shown in Table 1. But in an extreme mainly cold or non-uniform environment, it would be useful to assess the local thermal sensation, allowing us to observe of which body part the thermal state have larger effects on the whole-body thermal sensation and the parameters of sleep quality, which would help to find solutions such as local thermal stimulations to improve sleep quality.

The present study observed that when asleep, the skin temperature of the forehead was highly correlated with recalled thermal sensation (Table 2). Some studies have reported similar results for subjects who were awake: Taniguchi et al. (1992) observed that the average temperature of the skin of the face was closely related to whole-body thermal sensation votes [30]; Cotter and Taylor reported that the face was a highly thermosensitive area for both perceptual thermal sensitivity and autonomic sensitivity, compared to other locations across the body [27]. Other studies reported that the face was the most thermally sensitive area whereas the extremities had poor thermal comfort sensation when subjects were awake [31-33]. In addition, although thermal sensitivity declines with age in all locations, the thermal sensitivity of the forehead maintained in elderly people [34]. With regard to measurement reliability, it was observed that 
skin temperature sensors could remain attached to the forehead area for almost the entire sleeping period. The present results suggest that the skin temperature of the forehead is a good index of thermal state for subjects sleeping in a thermally uniform environment. It has even been suggested that skin temperature is better than room temperature if the aim is to control the indoor environment according to occupants' thermal perception [35]. In a sleeping environment, it would be particularly appropriate to control the environment based on the occupant's thermal state, because the body passes through different physiological and biochemical states during different periods of the night [9]. However, there were no really cold condition in our four experiments. When heavy bed coverings are required the body is exposed to extremely asymmetrical conditions, so the situation may be expected to be different in cold conditions. In a recent study, the effect of three different quilts on sleep quality and thermal comfort were investigated in a cold environment (at an air temperature of $11.5^{\circ} \mathrm{C}$ ) [36]. The skin temperature of the forehead did not change significantly, while those at chest and foot showed significant difference between the quilts. However, no significant difference in thermal sensation was observed, although compared with a cotton quilt, the subjects felt slightly warmer when a goose down quilt was used. Whether the skin temperature of the forehead is a good index of thermal comfort sensation in very cold environment remains to be investigated.

When sleeping, one part of the body is in contact with the bed and the other is not [37]. The former loses less heat to the environment, and consequently has a higher mean skin temperature [37]. The skin temperatures of the back and neck were found to be about $0.5^{\circ} \mathrm{C}$ higher than those of the forehead and chest when the subjects lay supine on a bed with no covering at an air temperature of $30^{\circ} \mathrm{C}$ [38]. The three sites used in the present three-point method are unlikely to be in contact with the bed, so the measured skin temperature is not the true MST, but a prediction of the thermal state experienced by a sleeping person. It is difficult to include the body parts that are intermittently in contact with the bed in an estimate of MST since body posture is variable during sleep and between people, and while it is necessary to do so in attempting to calculate whole-body heat loss, the present results indicate that it is not necessary when attempting to predict thermal sensation. They suggest that subjects take for granted that body parts in contact with the bed have a higher skin temperature, as was indeed observed in one of 
the experiments.

It has previously been reported that the thermally neutral temperature may be different while sleeping [9]. In daily life, the thermal environment selected for the bedroom may often be based on thermal sensation while awake in a pre-sleep period. This may explain why so many people wake up feeling cold even when they set the air conditioner according to their preference [3]. To achieve good sleep, it is preferable to control the thermal environment in the bedroom according to the thermal state that occurs when asleep. One possible approach would be to control the thermal environment in the bedroom based on skin temperature [35, 39]. Three local skin temperatures from different body segments have been shown to contain enough information to identify the steady-state thermal requirements of subjects who were awake [39]. More skin locations are not necessary [39]. As skin temperatures are more evenly distributed when sleeping, the MST calculated with the present three-point method could be expected to provide useful information for better control of the thermal environment in bedrooms. Since it may be possible to remotely measure the uncovered skin temperature at the forehead of a sleeping subject, using a thermal camera, the present finding that the skin temperature of the forehead predicted the thermal sensation of subjects who were asleep offers a real possibility of doing so. However, further experiments with human subjects are required to determine the viability of intelligent control of bedroom temperatures based on skin temperatures. It should also be noted that, the inter-personal variance in the skin temperature could be higher than $0.5^{\circ} \mathrm{C}$ (Figure 5,6), although it is slightly lower than those observed in subjects who were awake [40]. When using skin temperatures as the only input to intelligent control of the environment, machine learning could be used to derive the reference skin temperature for the individual. Individual differences, whose effects are eliminated by the design of within-subject experiments such as those considered here, could then be offset more efficiently.

\section{Conclusions}

- Skin temperatures were more evenly distributed across the body surface of sleeping subjects than has been reported for subjects who were awake. Under neutral conditions, the 
MST of sleeping subjects was approximately $34.6^{\circ} \mathrm{C}$, which was $1{ }^{\circ} \mathrm{C}$ higher than that of subjects who were awake.

- The skin temperature of the forehead was highly correlated with thermal sensation and could be obtained conveniently throughout 8 -hour exposures, so it is recommended as a good index of the thermal state of the body while sleeping, except for very cold environments in which the body is exposed to very asymmetrical conditions while sleeping.

- A three-point weighting method is proposed for estimating the MST of sleeping subjects from three measurement locations: the forehead, chest, and foot. The MST calculated with the three-point method was found to predict thermal sensation while sleeping better than MST calculated using the seven-point method attributed to Hardy and Dubois. Because of this, its convenience and reliability, and because it is less likely to disturb sleep, this threepoint method is recommended for obtaining the MST of sleeping subjects when the purpose is to predict their thermal sensation.

\section{Acknowledgement}

This work was supported by the National Natural Science Foundation of China (No. 51778359 and 51478260) and the State Key Laboratory of Air Conditioning Equipment and System Energy Conservation (No. ACSKL2018KT04).

\section{References}

[1] M.R. Opp. Sleeping to fuel the immune system: mammalian sleep and resistance to parasites, BMC Evol Biol. 9 (2009) 8-10.

[2] K. Okamoto-Mizuno, K. Mizuno. Effects of thermal environment on sleep and circadian rhythm, J Physiol Anthropol. 31 (2012) 14.

[3] L. Lan, K. Tsuzuki, Y.F. Liu, Z.W. Lian, Thermal environment and sleep quality: A review, Energy Build. 149 (2017) 101-113.

[4] K. Okamoto-Mizuno, K. Tsuzuki, K. Mizuno. Effects of mild heat exposure on sleep stages and body temperature in older men. Int J Biometeoral. 49 (2004) 32-36.

[5] E.J.W. Van Someren. More than a marker: interaction between circadian regulation of 
temperature and sleep, age-related changes, and treatment possibilities. Chronobiol Int, 17 (2000) 313-354.

[6] H. Zhang. 2003. Human thermal sensation and comfort in transient and non-uniform thermal environments. PhD Thesis. University of California, Berkeley.

[7] E.J.W. Van Someren. Sleep propensity is modulated by circadian and behavior-induced changes in cutaneous temperature. J Therm Biol. 29 (2004) 437-444.

[8] R.J.E Raymann, D.F. Swaab, E.J.W. Van Someren. Cutaneous warming promotes sleep onset. Am J Physiol Regul Integr comp Physiol. 288 (2007) R1589-R1597.

[9] L. Lan, Z.W. Lian, H.Y. Huang, Y.B. Lin, Experimental study on thermal comfort of sleeping people at different air temperatures, Build. Environ. 73 (2014) 24-31.

[10] K. Okamoto-Mizuno, K. Mizuno, S. Michie, A. Maeda, S. Iizuka, Effects of humid heat exposure on human sleep stages and body temperature, Sleep 22 (1999) 767-773.

[11] C.E. Winslow, A. L. P. Herrington, A. P. Gagge, A new method of partitional calorimetry. Am. J. Physiol. 116 (1936) 641-655.

[12] J. Hardy, E. Dubois, The technic of measuring radiation and convection, J. Nutr. 15 (1938) $461-475$.

[13] D.A. McIntyre. Indoor climate. London: Applied Science Publishers; 1980.

[14] H.Y. Wang, B. W. Olesen, O. B. Kazanci. Using thermostats for indoor climate control in offices: The effect on thermal comfort and heating/cooling energy use. Energy Build. 188-189 (2019) 71-83.

[15] D.Y. Lai, W.Y. Liu, T.T. Gan, K.X. Liu, Q.Y. Chen. A review of mitigating strategies to improve the thermal environment and thermal comfort in urban outdoor spaces. Sci. Total Environ. 661(2019) 337-353.

[16] C. Huizenga, H. Zhang, E. Arens, D. Wang. Skin and core temperature response to partialand whole-body heating and cooling. J Therm. Biol. 29 (2004) 549-558.

[17] N.L. Ramanathan, A new weighting system for mean surface temperature of the human body, J. Appl. Physiol. 19 (1964) 531-522.

[18] W.W. Liu, Z.W. Lian, Q.H. Deng, Y.M. Liu. Evaluation of calculation methods of mean skin temperature for use in thermal comfort study, Build. Environ. 46 (2011) 478-488.

[19] D. Mitchell, C.H. Wyndham, Comparison of weighting formulas for calculating mean skin 
temperature, J. Appl. Physiol. 26 (1969) 616-622.

[20] P.O. Fanger, Thermal comfort, Robert E Krieger Publishing Company, Malabar, 1982.

[21] H.Y. Wang, S.T. Hu. Analysis on body heat losses and its effect on thermal sensation of people under moderate activities, Build. Environ. 142 (2018) 180-187.

[22] L. Lan, Z.W. Lian, X.L. Qian, C.Z. Dai, The effects of programmed air temperature changes on sleep quality and energy saving in bedroom, Energy Build. 129 (2016) 207-214.

[23] L. Lan, Z.W. Lian, Y.B. Lin, Comfortably cool bedroom environment during the initial phase of the sleeping period delays the onset of sleep in summer, Build. Environ. 103 (2016) $36-43$.

[24] L. Lan, X.L. Qian, Z.W. Lian, Y.B. Lin, Local body cooling to improve sleep quality and thermal comfort in a hot environment, Indoor Air 281 (2018) 35-145.

[25] B.W. Olesen, How many sites are necessary to estimate a mean skin temperature, New York, Raven Press, 1984.

[26] Y. Houdas, E.F.J. Ring, Human body temperature: its measurement and regulation, New York, London, Plenum Press, 1982.

[27] J.D. Cotter, N.A. Taylor, The distribution of cutaneous sudomotor and alliesthesial thermosensitivity in mildly heat-stressed humans: an open-loop approach, J. Physiol. 565 (2005) $335-345$.

[28] K. Kräuchi, C. Cajochen, E. Werth, A. Wirz-Justice, Warm feet promote the rapid onset of sleep, Nature 401 (1999) 36-37.

[29] X. Zhou, Z.W. Lian, L. Lan. An individualized human thermoregulation model for Chinese adults. Build. Environ. 70 (2013) 257-265.

[30] Y. Taniguchi, H. Aoki, K. Fujikake, H. Tanaka, M. Kitada, Study on car air conditioning system controlled by car occupants' skin temperatures - Part 1: Research on a method of quantitative evaluation of car occupants' thermal sensations by skin temperatures, SAE Technical Paper Series 920169 (1992) 13-19.

[31] L.I. Crawshaw, E.R. Nadel, J.A. Stolwijk, B.A. Stamford, Effect of local cooling on sweating rate and cold sensation, Pflu“ gers Arch. 354 (1975) 19-27.

[32] J.C. Stevens, K.K. Choo, Temperature sensitivity of the body surface over the life span, Somatosens Mot. Res. 15 (1998) 13-28. 
[33] J.C. Stevens, L.E. Marks, D.C. Simonson, Regional sensitivity and spatial summation in the warmth sense, Physiol. Behav. 13 (1974) 825-836.

[34] Y. Inoue, N. Gerrett, T. Ichinose-Kuwahara, Y. Umino, S. Kiuchi, T. Amano, H. Ueda, G. Havenith, N. Kondo, Sex differences in age-related changes on peripheral warm and cold innocuous thermal sensitivity, Physiol. Behavior 164 (2016) 86-92.

[35] F. Wang, Z. Chen, Q. Feng, Q. Zhao, Z. Cheng, Z. Guo, Z. Zhong. Experimental comparison between set-point based and satisfaction based indoor thermal environment control, Energy Build. 128 (2016) 686-696

[36] M.C He, Z.W. Lian, P. Chen. Evaluation on the performance of quilts based on young people's sleep quality and thermal comfort in winter. Energy Build 183 (2019) 174-183.

[37] L. Lan, Z.Q. Zhai, Z.W. Lian. A two-part model for evaluation of thermal neutrality for sleeping people. Build. Environ. 132(2018) 319-326.

[38] X.L. Qian, L. Lan, Z.W. Lian. Effect of local cooling on thermal comfort of people in a sleeping posture. Procedia Engineering 205 (2017) 3277-3284.

[39] C.Z. Dai, H. Zhang, E. Arens, Z.W. Lian. Machine learning approaches to predict thermal demands using skin temperatures: Steady-state conditions. Build. Environ. 114 (2017) 1-10.

[40] L. Lan, Z. W. Lian, W. W. Liu, Y. M. Liu. Investigation of gender difference in thermal comfort for Chinese people. Eur J Appl. Physiol. 102 (2008) 471-480. 